\title{
On the formation mechanism of impurity-helium solids: evidence for extensive clustering
}

\author{
E.A. Popov ${ }^{1,2}$, J. Eloranta, ${ }^{1}$ J. Ahokas ${ }^{1}$, and H. Kunttu ${ }^{1}$ \\ ${ }^{1}$ Department of Chemistry, University of Jyväskylä, P.O.Box 35, FIN-40014, Finland \\ E-mail: Henrik.Kunttu@jyu.fi \\ ${ }^{2}$ Institute of Energy Problems of Chemical Physics, Russian Academy of Sciences \\ Chernogolovka, Moscow Region 142432, Russia
}

Received December 19, 2002

\begin{abstract}
Optical emission studies on a discharged nitrogen - helium gas jet injected into superfluid helium near $1.5 \mathrm{~K}$ are described. The analysis of atomic ( $\alpha$-group) and molecular Vegard-Kaplan transitions clearly indicates that the emitting species are embedded in the nitrogen clusters. The formation of the clusters is most efficient in the crater formed on the liquid surface. The model calculations based on the classical bubble model and density functional theory suggest that under the experimental conditions only clusters consisting of more than 1000 molecules have a kinetic energy sufficient for the stable cavity formation inside liquid helium. The results obtained suggest that the formation of impurity - helium solids is a consequence of extensive clustering in the gas jet.
\end{abstract}

PACS: 67.80.-s, 61.46.+w

\section{Introduction}

The experimental approach for stabilization of atoms by injection of impurity - helium ( $\mathrm{Im}-\mathrm{He})$ gas jet into superfluid helium (He II) was first developed by Gordon, Mezhov-Deglin, and Pugachev in 1974 [1]. The advantages of this approach are related to efficient pre-cooling of the gas jet prior to its immersion into He II, high degree of dispersion of impurity particles, and efficient thermal dissipation by He II. Consequently, stabilization of reactive atoms $(\mathrm{N}, \mathrm{H}, \mathrm{D})$ with exceedingly high densities has been achieved as indicated by optical emission and Electron Paramagnetic Resonance (EPR) measurements [2,3]. Since its discovery, the original approach has been subject to active development, and currently semitransparent gel-like substances with $\mathrm{He} / \mathrm{Im}$ ratios of $12-60$ and thermal stability up to $6-8 \mathrm{~K}$ are routinely grown. Although their interior is filled with liquid He, these macroscopic condensates are historically called impurity-helium solids (IHS) [1-9]. An interesting extension to the cited series of investigations is provided by a recent work by Mezhov-Deglin and Kokotin on the helium - water condensate [10].
Studies on thermal properties of IHS have shown that in presence of $\sim 0.5 \%$ mole fraction of impurity $\left(\mathrm{N}_{2}, \mathrm{Kr}\right)$ in the condensate completely suppresses the convection flow of liquid He filling the condensate $[6,9]$. It was supposed therefore, that IHS comprise a porous network structure and thus resemble highly porous aerogels filled with liquid He. More recently, strong support for this hypothesis has been provided by a series of structural studies by the Lee group at Cornell University. Based on the complementary use of ultrasound and x-ray diffraction techniques it has been shown that $\mathrm{Im}-\mathrm{He}$ solids are, indeed, mesoporous with characteristic impurity cluster size near $6 \mathrm{~nm}$, average impurity density $\sim 10^{20} \mathrm{~cm}^{-3}$, and wide pore distribution ranging from 8 to $860 \mathrm{~nm}$ [11-13].

Although the physical characteristics of $\mathrm{Im}-\mathrm{He}$ solids have been rather extensively studied for years, their formation mechanism and structure are not understood on the molecular scale.

The model suggested by Gordon and Shestakov ascribed the formation of metastable Im-He condensates to a Im-He solid phase (IHSP) consisting of «sticking-together» Van der Waals impurity-helium clusters, $\operatorname{Im}(\mathrm{He})_{n}$, i.e., a bare impurity atom or molecule surrounded by solid layers of He atoms in a 
superlattice-like arrangement $[4,14]$. This is, of course, a very hypothetical model, which for example neglects entirely the role of impurity clusters in formation of these solids.

The present study aims at providing new insights into our understanding of $\mathrm{Im}-\mathrm{He}$ condensates, and in particular their formation mechanism. Instead of interrogating the solids themselves, we concentrate in processes taking place in the $\mathrm{Im}-\mathrm{He}$ gas jet from the discharge zone to its final immersion into He II. Here the following issues are addressed: (i) the extent of cluster formation in the gas jet, (ii) interaction of impurity particles (bare atoms or molecules, small clusters) with liquid helium surface, and (iii) factors controlling their probability to penetrate into bulk liquid helium in the experimental conditions. In what follows we describe our spectroscopic observations in nitrogen-helium gas jets and present results from model calculations of solvation of nitrogen species in He II.

\section{Experimental methods}

The experimental setup used in the present study consists of a liquid He bath cryostat fitted with a set of quartz windows (Fig. 1). The inner diameter of the He bath is $120 \mathrm{~mm}$, and it accumulates $7 \mathrm{~L}$ of liquid $\mathrm{He}$, which allows operation for more than $12 \mathrm{~h}$. By pumping the helium reservoir with a one-stage mechanical pump $(20 \mathrm{~L} / \mathrm{s})$ the lowest accessible temper-

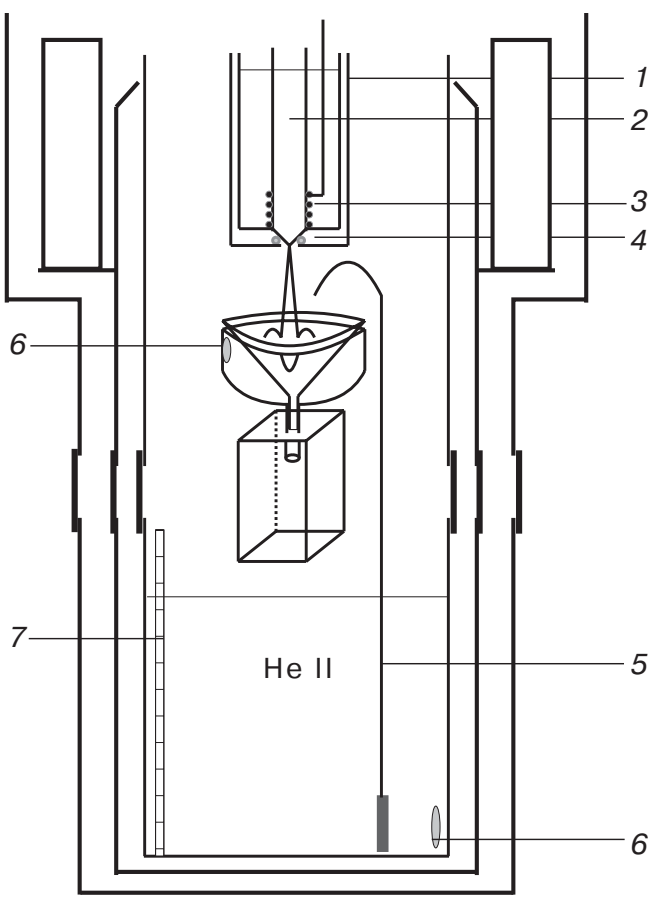

Fig. 1. Experimental setup for injection of $\mathrm{Im}-\mathrm{He}$ mixtures into superfluid helium: cryogenic discharge source (1); quartz tube (2); liquid nitrogen cooled inductance coil (3); nozzle (4), fountain pump (5); temperature sensors (6); He II level gauge (7). ature is near $1.4 \mathrm{~K}$. The vapor pressure of He inside the cryostat was measured with an absolute pressure transducer (MKS Instruments, Baratron model 622). The temperature was measured with a silicon diode sensor and Lake Shore 330 temperature controller.

A cryogenic discharge source, an optical cell and temperature sensors were placed inside the cryostat with an insertion allowing mutually independent operation of the instrumentation. This design of the insertion allows adjustment of the separation between the discharge tube orifice and the He surface in the sample cell in the range of $2-20 \mathrm{~cm}$. The electrodeless discharge was excited by an inductive coil, coaxially installed around a liquid nitrogen cooled quartz discharge tube $2 \mathrm{~cm}$ from the nozzle. The output of a home made pulse generator, operating near $40 \mathrm{MHz}$, was coupled to the coil, thus providing a RF discharge with a power $10-70 \mathrm{~W}$, and pulse duration ranging from $1 \mu$ s up to continuous operation.

Nitrogen and helium gases of 99.99(9)\% nominal purity were premixed in a stainless steel cylinder. A mechanical membrane regulator was used to provide constant gas flow with accuracy better than $5 \%$ for a typical gas flow rate of $5 \cdot 10^{19}$ particles/s upon pressure drop in the gas cylinder from 5 bar to $0.2 \mathrm{bar}$. The optical measurement cell consists of a $40 \mathrm{~mm}$ diameter quartz funnel attached to a standard quartz $10 \times 10 \mathrm{~mm}$ optical cuvette. Constant level of He II in the sample cell was maintained by a fountain pump, which supplied superfluid helium from the bottom of the cryostat.

The experiments were performed by passing a mixture of molecular nitrogen, diluted to $0.3-3 \%$ by helium gas, through the discharge zone. The discharged gas then escapes through a $0.8 \mathrm{~mm}$ diameter nozzle, and propagates through the dense cold helium gas, forming an intensively illuminated jet. The jet proceeds to the surface of He II producing a visually observable crater. Special attention was paid for selective collection of emission from three distinct observation zones, namely the gas jet, the crater, and bulk He II. For this purpose in some experiments the quartz funnel was blocked with a black painted Pyrex cover in order to allow collection of light solely from bulk He II.

The collected light was focused onto the entrance slit of a $0.3 \mathrm{~m}$ spectrograph (Acton), equipped with 2400, 600, 600 lines / $\mathrm{mm}$ gratings, blazed at 240,300, $500 \mathrm{~nm}$, respectively. The spectra were recorded with a thermoelectrically cooled Charge-Coupled-Device (CCD) camera (Princeton Instrument) attached to the spectrograph. Depending on the grating, the spectral bandwidth on the detector was 30,120, or 120 $\mathrm{nm}$, respectively. For more selective collection of light 
from different parts of the jet or bulk He II, a high-grade UV quartz optical fiber bundle was used.

\section{Experimental results}

\subsection{Characteristics of the jet}

After passing the RF discharge zone, the nitrogen-helium gas mixture propagates in dense cold helium gas (density $3 \cdot 10^{19}$ atoms per $\mathrm{cm}^{3}, T=1.7 \mathrm{~K}$ ) in a laminar mode, forming a well-collimated jet (Fig. 2). The peripheral part of the jet is rather cold, but its core is relatively hot and less luminescent. We have measured the velocity of the jet at $T=77 \mathrm{~K}$ and at cryostat pressure of 5 mbar. The measurement was performed by adjusting the discharge to $200 \mu$ s pulse and $500 \mathrm{~Hz}$ repetition rate. Under these conditions emission was monitored at $5 \mathrm{~cm}$ distance from the discharge tube orifice with a photomultiplier tube fitted with a horizontal $1.5 \mathrm{~mm}$ slit. From the observed

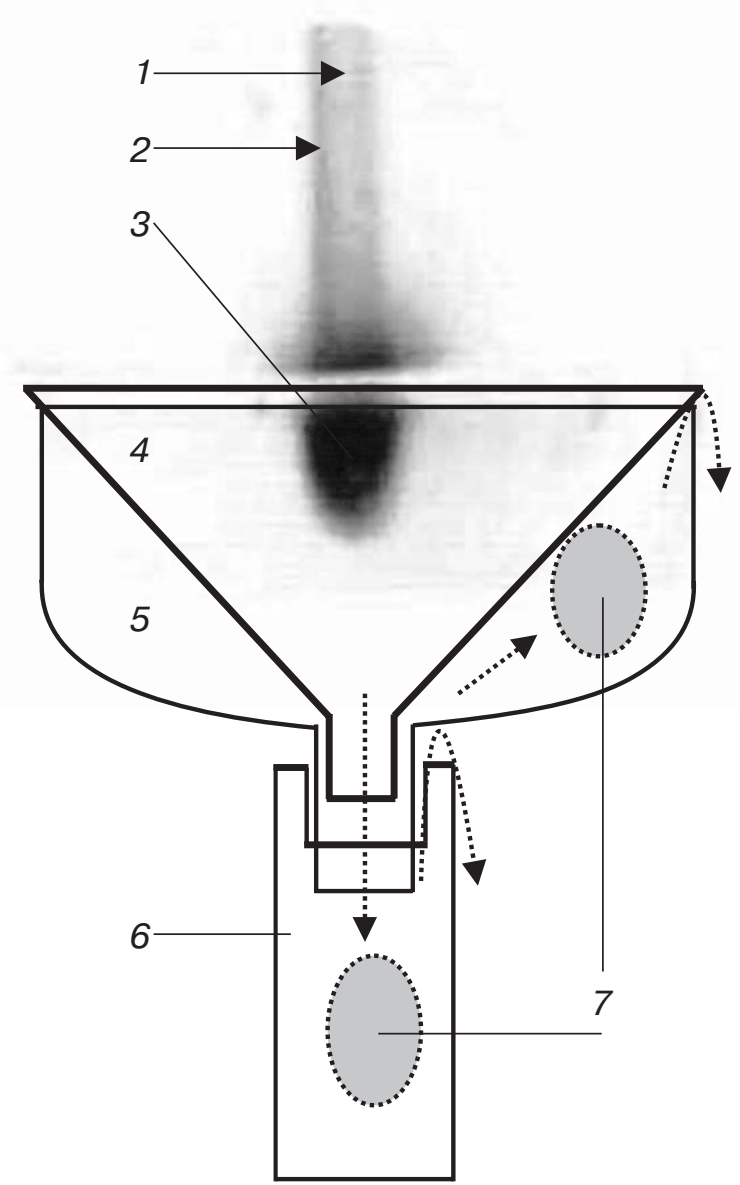

Fig. 2. A photograph of nitrogen-helium jet penetrating into bulk He II. A schematic view of the optical cell is also shown: the core of the jet (1); peripheral part of the jet (2); crater (3); black painted Pyrex funnel (4); quartz funnel (5); quartz cuvette (6); light collection zones (7). Dotted arrows show the circulation of liquid He. phase shift with respect to the pulse train, the flow velocity was estimated to be $v=70 \mathrm{~m} / \mathrm{s}$.

While hitting the He II surface the jet stops and produces a well defined crater. The crater is the most intensively emitting region due to effective collisional processes, which in turn promote aggregation of impurities, and recombination of nitrogen atoms. The intensity of the emission decreases dramatically in the bulk He II, even at distances few $\mathrm{mm}$ beneath the crater. Upon increasing the distance between the nozzle and the He II surface to $10 \mathrm{~cm}$ or more, we observed a transition from laminar (upper part) to purely turbulent flow (lower part), which is visually characterized by shaggy shape of the lower part of the jet.

\subsection{Emission spectra}

The main monitored emissions were the atomic $\mathrm{N}$ $\left({ }^{2} D^{-4} S\right)$ transition ( $\alpha$-group, green afterglow), as well as the Vegard-Kaplan $A^{3} \Sigma_{u}^{+}-X^{1} \Sigma_{g}^{+}$afterglow in blue and UV range) system of molecular nitrogen. Both of these transitions are forbidden in the gas phase and thus serve as sensitive probes for various processes related to the formation of impurity clusters and IHS.

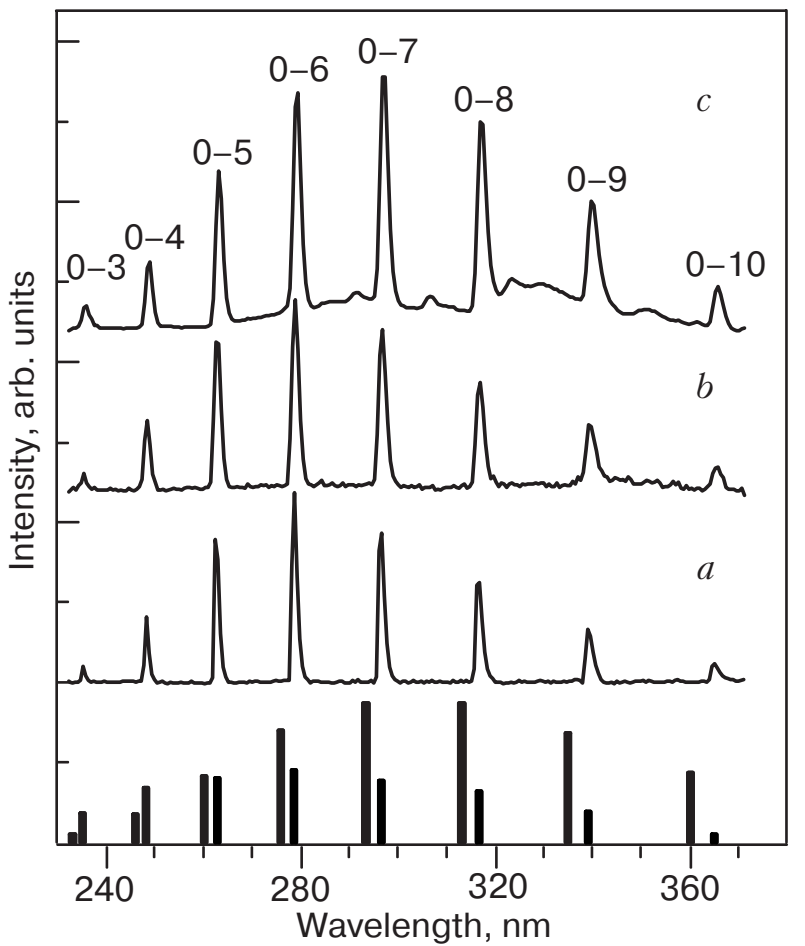

Fig. 3. A section of the Vegard - Kaplan emission band of molecular nitrogen collected from the gas jet $(a)$, bulk He II $(b)$, and upon explosion of the nitrogen-helium solid $(c)$. The numbers refer to the quantum labels $\left(v^{\prime}-v^{\prime \prime}\right)$. The stick spectra show the line positions in the gas phase (black columns, Ref. 17), and in solid nitrogen matrix (gray columns, Ref. 18). 
A section of the the Vegard - Kaplan band of $\mathrm{N}_{2}$ is presented in Fig. 3. The spectra are almost identical, regardless of the location of the emitter (gas jet, crater, bulk), and are characterized by linewidths of $\sim 1 \mathrm{~nm}$, and a consistent red shift of $\sim 360 \mathrm{~cm}^{-1}$ from the corresponding gas phase lines. As described in the experimental section, special attention was paid to eliminate stray light from other parts of the cryostat. Although spectroscopy of IHS is outside the scope of this report, a spectrum obtained from explosion of a helium-nitrogen condensate is provided for reference. Apart from the broad, so far unassigned background features, this spectrum clearly resembles the others. These observations would strongly suggest that radiative recombination of nitrogen atoms, leading to Vegard - Kaplan band, occurs in very similar environments in all detection zones.

The strongly forbidden green $\alpha$-group emission of atomic nitrogen was detected even in the gas jet, far from the liquid helium surface. The $\alpha$-group emission, collected near the crater was very intense and the spectrum contained three peaks, centered at 521 and 522, and $523 \mathrm{~nm}$ (see Fig. 4). This observation pro-

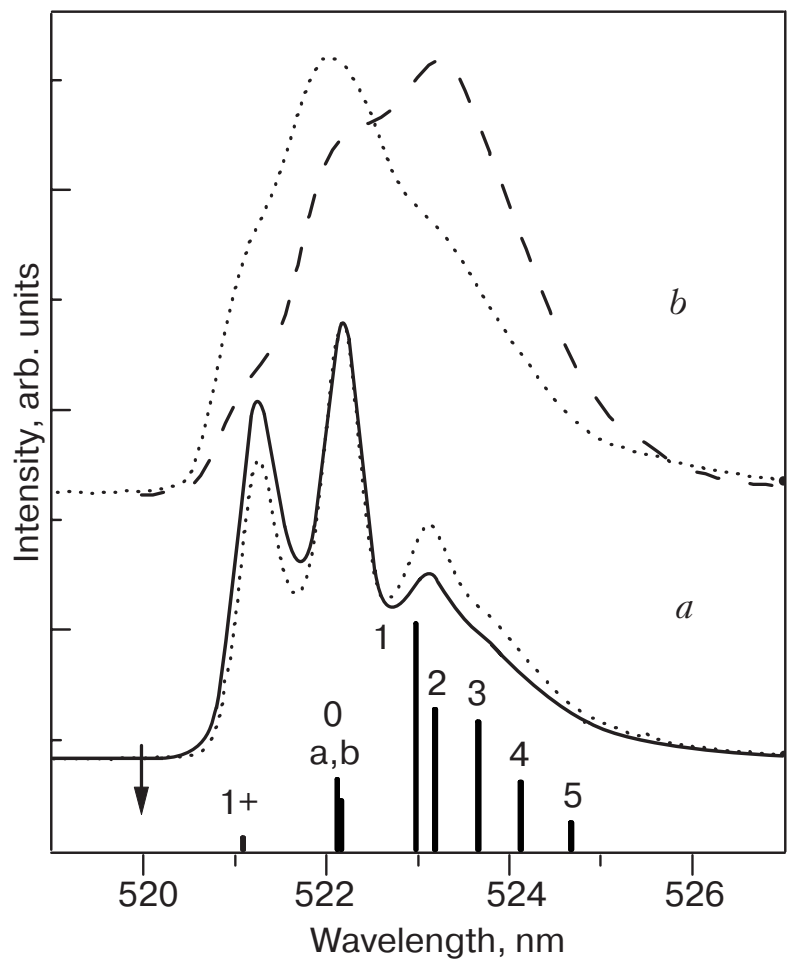

Fig. 4. The effect of the gas mixture on the ${ }^{2} D-{ }^{4} S$ emission of atomic nitrogen. $\mathrm{N}_{2} / \mathrm{He}=1 / 400$ (solid line), $1 / 100$ (dotted line). A grating with 2400 groves $/ \mathrm{mm}$ was used (a). $\mathrm{N}_{2} / \mathrm{He}=1 / 100$ (dotted line), 1/30 (dashed line). Grating with 600 groves/mm was used $(b)$. The arrow indicates the gas phase position of the transition (Ref. 19). The stick spectrum represents observation in solid nitrogen matrix (Ref. 24). vides additional support for the assignment that, instead of monitoring transitions of more or less isolated gas phase species, the spectra are strongly affected by formation of clusters in the gas jet before it enters in He II. No significant changes in the $\alpha$-group emission was observed when signal was collected from bulk $\mathrm{He}$ II. Increasing the $\mathrm{N}_{2} / \mathrm{He}$ ratio of the gas mixture from 0.3 to $3 \%$, caused the shift of the $\alpha$-group emission towards the $523 \mathrm{~nm}$ in the gas phase. We performed spectroscopic studies on other atomic and molecular transitions such as $\left.\mathrm{N}^{2} P{ }^{2} D\right)$, the first positive system $\left(B^{3} \Pi-A^{3} \Sigma\right)$ of $\mathrm{N}_{2}, \mathrm{O}\left({ }^{1} S-{ }^{1} D\right)$, the Herzberg I bands $(A-X)$ of $\mathrm{O}_{2}$, as well as the $\beta, \gamma$, and $\delta$ bands of NO. These data, which will be published separately, are consistent with the present observations.

\section{Discussion}

In the present study we concentrate on the Vegard-Kaplan $(\mathrm{V}-\mathrm{K})$ emission system in the UV region mainly for two reasons: (i) previous spectroscopic measurements in IHS were restricted to the visible range [5] and (ii) we expected to observe recombination of atomic nitrogen in bulk He II and in nitrogen - helium solid by monitoring radiative decay of the metastable $A^{3} \Sigma$ state of the $\mathrm{N}_{2}$ molecule (triplet exciton in the case of IHS). Detection of the $\mathrm{V}-\mathrm{K}$ emission in the gas phase is a rather challenging task and would necessitate very high purity gases, especially free of any oxygen-containing impurities. Moreover, the discharge needs to be operated under special conditions [15,16]. Quite surprisingly, we observed rather intense $\mathrm{V}-\mathrm{K}$ emission from the gas jet even in the presence of oxygen impurities. Equally demonstrative is the observation that the $\mathrm{V}-\mathrm{K}$ lines are red-shifted with respect to their pure gas phase counterparts [17]. This would, indeed, strongly suggest that the emission originates from recombination of atoms embedded in impurity particles. The $\mathrm{V}-\mathrm{K}$ emission was studied in solid nitrogen matrix by Coletti and Bonnot [18]. They observed rather intense emission at $20 \mathrm{~K}$ with a lifetime of the order of $\sim 1 \mathrm{~ms}$. Similarly to our findings in the nitrogen - helium jet, the $\mathrm{V}-\mathrm{K}$ lines are red shifted from the gas phase by $350 \mathrm{~cm}^{-1}$ in solid nitrogen.

The atomic $\left({ }^{2} D_{5 / 2,3 / 2}-{ }^{4} S_{3 / 2}\right)$ transitions of nitrogen are strongly forbidden by spin and parity selection rules of electric dipole, and their observation is ascribed mostly to electric quadruple. The ${ }^{2} D_{5 / 2}$ and ${ }^{2} D_{3 / 2}$ states lie $19224 \mathrm{~cm}^{-1}$ and $19233 \mathrm{~cm}^{-1}(2.38 \mathrm{eV})$ above the ${ }^{4} S_{3 / 2}$ ground state, and their calculated lifetimes are $44 \mathrm{~h}$ and $17 \mathrm{~h}$, respectively [19]. Thus, detection of pure atomic emission within the time of flight of the jet should not be feasible at the con- 
ditions of the present experiments. Therefore, some process taking place in the gas jet is dramatically affecting the transition probability, and consequently decreasing the lifetime of the atomic transition. Aggregation of nitrogen molecules around the emitter would obviously be such a process. In fact, unlike in the gas phase, in an irradiated nitrogen solid the green atomic emission is the most prominent spectral feature [20]. In solid nitrogen this emission consists of eight main lines, the most intense being at $522 \mathrm{~nm}$ (zero-phonon line) and 523-525 nm (phonon-induced wing). Furthermore, the ${ }^{2} D-{ }^{4} S$ emission has a life time of $40 \mathrm{~s}$ in solid nitrogen [21]. The similarity between our spectra and the one obtained in solid nitrogen is obvious already at the highest dilution, and gets stronger at increased nitrogen content (see Fig. 5).

One important factor promoting nucleation and cluster formation in the jet is related to its ability to switch from laminar to turbulent mode upon lowering the temperature. Let us consider the jet as an incompressible flow through dense helium gas. Within this framework the Reinolds number is defined by the straightforward relation $R_{e}=v D / v$. Here $v$ is the velocity of the jet, $D$ is the diameter of the jet (pipe flow), and $v$ is the kinematic viscosity (viscosity divided by density). Upon decreasing the temperature of the nitrogen-helium jet
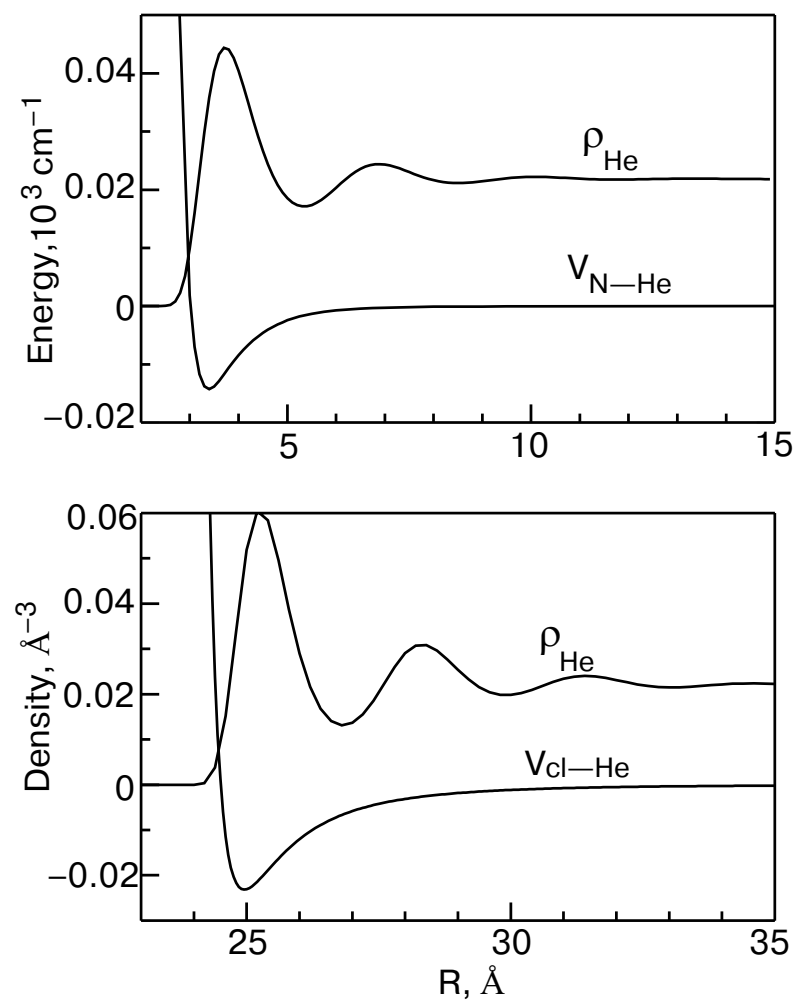

Fig. 5. The density profiles of liquid He near ground state nitrogen atom (upper panel) and molecular nitrogen cluster with $n=1000$ (lower panel) are shown. The corresponding pair potentials are also shown. from initial $T=80 \mathrm{~K}$ to $T=10 \mathrm{~K}$ (temperature few $\mathrm{mm}$ above the He II surface), the kinematic viscosity of the jet decreases by a factor of 25. In a pipe flow characterized by $v=70 \mathrm{~m} / \mathrm{s}, D=0.005 \mathrm{~m}, v(80 \mathrm{~K})=1.8 \cdot 10^{-3} \mathrm{~m}^{2} / \mathrm{s}$, $v(10 \mathrm{~K})=6 \cdot 10^{-5} \mathrm{~m}^{2} / \mathrm{s}$, the jet should change from lami$\operatorname{nar}\left(R_{e}=210\right.$ at $\left.T=80 \mathrm{~K}\right)$, to turbulent $\left(R_{e}=5600\right.$ at $T=$ $=10 \mathrm{~K})$. We observed indeed a shaggy front of the jet in the vicinity of He II surface. Moreover, the shaggy front was clearly seen even when the distance between He II surface and the nozzle was more than $10 \mathrm{~cm}$, and the jet did not reach the surface. We assign this observation to the onset of turbulent flow.

Visual observation during preparation of IHS showed that some small particles penetrate into bulk He II directly from the jet. However, most of the impurities tend to float on the surface and stick to the walls of the quartz cell, growing on the walls, and finally sink down to the bottom. In the following we assume that penetration of an impurity into liquid $\mathrm{He}$ depends on the kinetic energy of the particle, which in the present case is defined by the velocity of the propagating jet. More precisely, the kinetic energy should exceed the energy required for solvating the given species. This energy can be estimated by the classical bubble model:

$$
E_{b}\left(R_{b}\right)=V_{\mathrm{N}-\mathrm{He}}\left(R_{b}\right)+4 \pi R^{2}{ }_{b} \gamma
$$

where $R_{b}$ is the bubble radius and $\gamma$ is surface tension $\left(\gamma_{\mathrm{He}}=3.6 \cdot 10^{-4} \mathrm{~J} / \mathrm{m}^{2}\right)$. By neglecting all dissipative processes, and the van der Waals attraction, one can obtain a lower bound for the critical velocity required for a species to penetrate into the liquid:

$$
v_{\text {cr }}>4 R_{b}(\pi \gamma / m)^{1 / 2}
$$

In order to estimate the radius of the cavity produced by a solvated cluster we need to evaluate the interaction potential of the cluster in liquid helium. Here we rely on the approximate $\mathrm{N}_{2}-\mathrm{He}$ Lennard-Jones (LJ) potential with $\varepsilon=18 \mathrm{~cm}^{-1}$ and $R_{0}=3.6 \mathrm{~A}$ [22]. By assuming a perfect spherical cluster with $N=1000$, sharp edge, and $\rho=1 \mathrm{~g} / \mathrm{cm}^{3}=0.0217$ molecules $/ \mathrm{A}^{3}$ we end up with a radius of $22.2 \mathrm{~A}$. The effective interaction potential can be formulated as:

$$
V_{\mathrm{eff}}(r)=\int \rho_{\mathrm{N}_{2}}\left(r^{\prime}\right) V_{L J}\left(\mid r-r^{\prime}\right) d^{3} r^{\prime} .
$$

We computed solvation of impurity $\mathrm{N}\left({ }^{2} D\right)$ and $\mathrm{N}\left({ }^{4} S\right)$ atoms as well as the $\left(\mathrm{N}_{2}\right)_{1000}$ cluster in liquid helium using density functional theory [23]. Figure 5 represents results of such calculation for a bare $\mathrm{N}$ atom and a $\left(\mathrm{N}_{2}\right)_{1000}$ cluster in a spherical cavity. We can now estimate the critical velocities for a single atom $\left(R_{b}=\right.$ $=4 \AA)$ and the cluster $\left(R_{b}=22.2 \AA\right)$. Substitution into Eq.(1) yields $v_{\mathrm{N}}>400 \mathrm{~m} / \mathrm{s}$ and $v_{\mathrm{cl}}>70 \mathrm{~m} / \mathrm{s}$ 
for the atom and the cluster, respectively. It should be emphasized here that we neglect all other dissipative processes such as creation of ripplons and shock waves. Consequently, the real values should be even greater. On the other hand, Van der Waals binding should slightly favor solvation. Although our theoretical treatment is relatively crude, it clearly shows that under the experimental conditions, seeding bare atoms into bulk helium is not feasible by a slowly propagating gas jet.

\section{Summary}

We have described optical emission studies on discharged nitrogen - helium gas mixtures under experimental conditions in which IHS are typically prepared. The analysis of the observed atomic ( $\alpha$-group) and molecular Vegard-Kaplan transitions clearly indicate that, instead of isolated atoms or molecules, these emissions originate from nitrogen clusters or matrix-like particles. The formation of clusters takes place already in the discharge zone, and is most efficient at the point where the gas jet hits the surface of He II, and a well defined crater is formed. The enhancement of emission intensity at the lower part of the jet is ascribed to change from laminar to turbulent flow.

Penetration of chemical species into liquid He II is discussed within the classical bubble model. The calculated critical velocity, $400 \mathrm{~m} / \mathrm{s}$, needed for solvation of a bare $\mathrm{N}$ atom clearly exceeds the measured velocity of the jet, and only clusters consisting of at least 1000 molecules may have sufficient kinetic energy to overcome the barrier for stable cavity formation in bulk He II. By combining the present experimental findings and model calculations, we conclude that formation of impurity clusters, i.e., building blocks of IHS, occurs via extensive clustering in the jet and, most efficiently, in the crater. Coalescence of these nanosize clusters inside He II leads then to formation of macroscopic condensates. Finally, deposition of impurity - helium jet into the liquid helium through its surface could be utilized for efficient formation of mass-selected neutral clusters and production of amorphous materials. Aerogel-like IHS, formed from $\mathrm{Ne}, \mathrm{Ar}, \mathrm{Kr}$, and $\mathrm{N}_{2}$ impurities represent one of such example.

\section{Acknowledgements}

We wish to thank David M. Lee and Vladimir Khmelenko for fruitful discussions on impurity helium solids. This work was supported by the Academy of Finland.
1. B. Gordon, L.P. Mezhov-Deglin, and O.F. Pugachev, JETP Lett. 19, 63 (1974).

2. E.B. Gordon, A.A. Pelmenev, O.F. Pugachev, and V.V. Khmelenko, JETP Lett. 37, 282 (1983).

3. E.B. Gordon, V.V. Khmelenko, A.A. Pelmenev, E.A. Popov, and O.F. Pugachev, Chem. Phys. Lett. 155, 301 (1989).

4. E.B. Gordon, V.V. Khmelenko, A.A. Pelmenev, E.A. Popov, O.F. Pugachev, and A.F. Shestakov, Chem. Phys. 170, 411 (1993).

5. R.E. Boltnev, E.B. Gordon, V.V. Khmelenko, I.N. Krushinskaya, M.V. Martynenko, A.A. Pelmenev, E.A. Popov, and A.F. Shestakov, Chem. Phys. 189, 367 (1994).

6. M.V. Martynenko, V.N. Novikov, A.A. Pelmenev, E.A. Popov, and E.V. Shidov, Bull. of Moscow Univer. 3, 53 (1996)

7. R.E. Boltnev, I.N. Krushinskaya, M.V. Martynenko, A.A. Pelmenev, E.A. Popov, and V.V. Khmelenko, Fiz. Nizk. Temp. 23, 753 (1997) [Low Temp. Phys. 23, 567 (1997)].

8. R.E. Boltnev, I.N. Krushinskaya, A.A. Pelmenev, D.Yu. Stolyarov, and V.V. Khmelenko, Chem. Phys. Lett. 305, 217 (1999).

9. E.A. Popov, A.A. Pelmenev, and E.B. Gordon, J. Low. Temp. Phys. 119, 367 (2000).

10. L.P. Mezhov-Deglin and A.M. Kokotin, J. Low Temp. Phys. 119, 385 (2000).

11. S.I. Kiselev, V.V. Khmelenko, D.A. Geller, D.M. Lee, and J.R. Beamish, J. Low Temp. Phys. 119, 357 (2000).

12. S.I. Kiselev, V.V. Khmelenko, and D.M. Lee, Fiz. Nizk. Temp. 26, 874 (2000) [Low Temp. Phys. 26, $641(2000)]$.

13. S.I. Kiselev, V.V. Khmelenko, D.M. Lee, V. Kiryukhin, R.E. Boltnev, and E.B. Gordon, Phys. Rev. B65, 24517 (2001).

14. E.B. Gordon and A.F. Shestakov, Fiz. Nizk. Temp. 26, 5 (2000) [Low Temp. Phys. 26 (2000)].

15. R.E. Miller, J. Chem. Phys. 43, 1695 (1965).

16. A.M. Pravilov, L.G. Smirnova, and A.F. Vilesov, Chem. Phys. Lett. 109, 343 (1984).

17. A. Loftus and K.H. Krupenie, J. Phys. Chem. Ref. Data 6, 113 (1977).

18. F. Coletti and A.M. Bonnot, Chem. Phys. Lett. 45, 580 (1977).

19. B.C. Fawsett, At. Data Nucl. Data Tables 16, 135 (1975).

20. A.M. Bass and H.P. Broida, Formation and Trapping of Free Radicals, Academic Press, New York (1960).

21. D.S. Tinti and G.W. Robinson, J. Chem. Phys. 49, 3229 (1968).

22. P. Habitz, K.T. Tang, and J.P. Toennies, Chem. Phys. Lett. 85, 461 (1982).

23. J. Eloranta, N. Schwentner, and V.A. Apkarian, J. Chem. Phys. 116, 4039 (2002).

24. O. Oehler, D.A. Smith, and K. Dressler, J. Chem. Phys. 66, 2097 (1977). 